Report

\title{
Is stereotactic large-core needle biopsy beneficial prior to surgical treatment in BI-RADS 5 lesions?
}

\author{
Lidewij E. Hoorntje ${ }^{1,2,3}$, Petra H.M. Peeters ${ }^{2}$, Willem P.T.M. Mali ${ }^{3}$, and Inne H.M. Borel \\ Rinkes ${ }^{1}$ \\ ${ }^{1}$ Department of Surgery; ${ }^{2}$ Julius Center for Health Sciences and Primary Care; ${ }^{3}$ Department of Radiology, \\ University Medical Center, Utrecht, The Netherlands
}

Key words: breast cancer, BI-RADS, nonpalpable, stereotactic large-core biopsy

\begin{abstract}
Summary
Introduction. Due to screening mammography, more nonpalpable mammographic lesions warrant histological evaluation. Stereotactic large-core needle biopsy (SLCNB) has been shown to be as effective in diagnosing these lesions as diagnostic surgical excision, and has become the preferred diagnostic procedure for most mammographic lesions. Since radiologically malignant BI-RADS 5 lesions are almost always carcinoma, some centers advocate prompt diagnostic surgical excision for these lesions instead of SLCNB. For some patients this diagnostic surgical intervention may serve as definitive treatment. We set out to find a subgroup of mammographic BI-RADS 5 lesions for which surgical biopsy might be preferable.

Methods. Of 1644 consecutive nonpalpable lesions referred for SLCNB between April 1997 and May 2002, 238 were classified as BI-RADS 5. We assessed the number of carcinomas and the surgical interventions performed. Outcomes were compared between various types of mammographic lesions: density with calcifications, density without calcifications, and calcifications only. Different theoretical strategies for diagnostic work-up of BI-RADS 5 lesions were explored.

Results. Carcinoma was found in 229/238 lesions (96\%). Most mammographic densities were invasive cancer (97\%), while calcifications only showed the highest risk for DCIS (51\%). In our study (current practice) all lesions were scheduled to first undergo SLCNB. A scenario was proposed where all lesions with only a density would be scheduled directly for sentinel node biopsy (SNB) and tumour excision $(n=154$; $65 \%$ ), while other lesions would still be scheduled for SLCNB. When we compared this scenario to current practice, four out of 238 patients $(<2 \%)$ would be 'overtreated' with SNB.

Conclusions. Our findings confirm a high predictive value of malignancy for BI-RADS 5 lesions (96\%). Surgical excision is therefore imperative for all BI-RADS 5 lesions, irrespective of SLCNB results. For BIRADS 5 lesions presenting as mammographic densities only, we propose to consider surgical excision with SNB to be the first diagnostic and therapeutic procedure. SLCNB is preferred in all other cases.
\end{abstract}

\section{Introduction}

Due to screening mammography there are increasingly more nonpalpable lesions referred for histological diagnosis. The minimally invasive stereotactic large-core needle biopsy has been shown to be equally effective in diagnosing these lesions as the diagnostic surgical excision biopsy [1]. The advantages of the minimally invasive needle technique (i.e., less morbidity, lower costs) have led to a situation where it is the diagnostic procedure of first choice for all nonpalpable breast lesions [2].

A proportion of all nonpalpable breast lesions will be classified as mammographically malignant, or BI-RADS 5. The Breast Imaging Reporting and Data System (BI-RADS) lexicon was developed by the American College of Radiology to standardize 
mammographic findings and assign a level of cancer suspicion to each finding, ranging from 1 (normal) to 5 (highly suggestive of malignancy) [3]. The risk of malignancy for each category has been well established [4]. BI-RADS 5 lesions have a very high risk of malignancy, with positive predictive values between 82 and 97\% [5-7]. In these cases, one might argue that large-core needle biopsy (LCNB) is a waste of time, since surgical excision will be necessary in all cases. Even when SLCNB shows a benign lesion, because of the discrepancy with the mammographic classification, the lesion will need to be removed [5,6,8]. Some centers therefore advocate diagnostic surgical excision for BI-RADS 5 lesions instead of SLCNB [9-12]. The advantage would be that for some patients the diagnostic surgical intervention may serve as definitive treatment, i.e. for those with radical excision of the lesion.

On the other hand, even though almost all BI-RADS 5 lesions are malignant, a distinction between in situ or invasive carcinoma is important in planning surgical treatment. For ductal carcinoma in situ (DCIS), wide local excision of the tumour is the standard treatment; axillary assessment is not necessary. With a preoperative diagnosis of invasive cancer, axillary sampling (i.e., sentinel node biopsy) at the time of the first surgical intervention should be planned in addition to tumourectomy. Based on SLCNB results, a distinction between in situ and invasive cancer can be made before planning the first operative procedure.

We hypothesized that for some BI-RADS 5 lesions, SLCNB does indeed not render additional value, and a (therapeutic) strategy can be safely planned based solely on the radiological characteristics. We assessed the number of carcinomas as well as the total number and type of surgical interventions performed on patients with BIRADS 5 lesions. In addition, we set out to find a subgroup of mammographic BI-RADS 5 lesions for which surgical biopsy might be the procedure of first choice.

\section{Materials and methods}

In this study, all 1644 consecutive nonpalpable lesions referred for stereotactic 14G-needle biopsy between April 1997 and May 2002 were classified in accordance with the BI-RADS assessment categories prospectively before biopsy. All BIRADS 5 lesions were included in the study $(n=238)$. Our protocol is described in detail elsewhere [1].

We assessed the number of carcinomas and, specifically, the number of invasive carcinomas, as well as the total number of surgical interventions performed. Surgical interventions were mastectomy or local excision with or without sentinel node biopsy (SNB). In addition, we compared these outcomes between the various types of mammographic lesions: density with calcifications, density without calcifications, and calcifications only.

We do not describe the materials used for the SNB ( i.e., which isotope and/or which blue dye), since we propose scenario's that may be applicable in various clinics, with various methods of SNB. We do want to underline however, that SNB, although minimally invasive, is not a risk-free operation: if patients are having lymphazurin blue dye there is a documented 1-2\% allergic reaction rate some of which have resulted in anaphylactic reactions and patient deaths. Even though the number is low this risk needs to be taken into account in practice but is not further discussed in this paper.

We propose two strategies for diagnostic workup (diagnosis and/or treatment) of BI-RADS 5 lesions and discuss (dis)advantages of each of these.

\section{Results}

Characteristics of the patients and lesions, as well as the performed surgical operations, are presented in Table 1 . In $14 \%$ of patients with a mammographic density more than one operation was necessary (21/154). These percentages were 33 and $29 \%$, respectively, for lesions consisting of a density with calcifications (14/43) and for calcifications only (12/41).

Table 2 shows predictive values for each of these three types of mammographic lesions. All three types show a high risk for breast cancer (overall, 96\%), but the risk for invasive cancer was highest in lesions presenting as a density, while calcifications only showed the highest risk for DCIS.

Next, we considered two theoretical scenarios. In scenario I all BI-RADS 5 lesions that present as a density (with or without calcifications) are 
Table 1. Patient- and lesion characteristics of 238 BI-RADS 5 lesions

\begin{tabular}{lcc}
\hline & $n(238)$ & $(\%)($ range $)(100)$ \\
\hline Age (median; years) & 62 & $(43-84)$ \\
Malignant & 229 & $(96)$ \\
$\quad$ Invasive cancer & $199^{\mathrm{a}}$ & $(84)$ \\
$\quad$ DCIS & 30 & $(13)$ \\
First operative procedure & 77 & \\
$\quad$ Mastectomy & 159 & $(32)$ \\
$\quad$ Local excision & 2 & $(67)$ \\
Sentinel node biopsy & 191 & $(1)$ \\
\# Operations & 45 & $(80)$ \\
1 & 2 & $(19)$ \\
2 & & $(1)$ \\
3 & 36 & $(77)$ \\
Reasons for $>$ operation $(n=47)$ & 8 & $(17)$ \\
close/positive margins & 2 & $(4)$ \\
axillary clearance after SNB + & 1 & $(2)$ \\
mastectomy after SNB & & \\
excision of infected seroma & & \\
\hline
\end{tabular}

${ }^{\mathrm{a}}$ In 8 cases SLCNB showed only DCIS; but subsequent surgical excision revealed invasive cancer.

scheduled directly for SNB and excision of the tumour. In our series, this would encompass 197 lesions, of which 181 (92\%) were invasive cancer, nine were DCIS and seven were nonmalignant. This would result in overtreatment for 16 of 238 patients (7\%), for whom SNB turned out to be not necessary because no invasive carcinoma was present. All lesions consisting of calcifications only would be planned for SLCNB, after which surgical therapy would be initiated. In scenario II all lesions with only a density $(n=154)$ are scheduled directly for SNB and tumour excision. Four out of 238 patients $(<2 \%)$ would turn out to have benign disease. All lesions with calcifications $(n=84)$ would first undergo SLCNB, followed by surgical therapy.

In short, compared to SLCNB for all lesions (current practice $=$ scenario 0 ), scenario I would prevent SLCNB in 197 out of 238 cases (83\%) but 16 of 238 patients would undergo unnecessary SNB. Scenario II would prevent 154 out of 238 SLCNB (65\%) but only four patients would undergo unnecessary SNB.

\section{Discussion}

Our findings confirm the high predictive value of malignancy for BI-RADS 5 lesions (96\%). Surgical excision is therefore imperative for all BI-RADS 5 lesions, even when SLCNB shows benign disease. In scenario II we adopt a scenario where all lesions presenting as mammographic densities without calcifications would be initially planned for sentinel node biopsy and local excision of the breast tumour in stead of SLCNB, $65 \%$ of SLCNB would be omitted, and $<2 \%$ of patients would be 'overtreated' with a SNB.

Our findings are comparable to results described by others: most BI-RADS 5 calcifications represent DCIS, whereas most BI-RADS 5 densities represent invasive cancer $[5,13]$. The preference for either of the scenario's we present obviously depends on the prevalence of invasive cancer and DCIS.

With regards to costs, the results of Fahy et al. do not support the preferential use of SLCNB or surgical biopsy for the evaluation of BI-RADS 5 lesions, since they may be evaluated by either method without a significant effect on total cost [2]. Lee et al. [14] did find small cost savings using SLCNB to diagnose BI-RADS 5 lesions; the percentage of malignancies in this group was $75 \%$. Gisvold et al. [11] found carcinoma in $82 \%$ of BIRADS 5 lesions and concluded that SLCNB is an unnecessary expenditure of time and money for such lesions. 


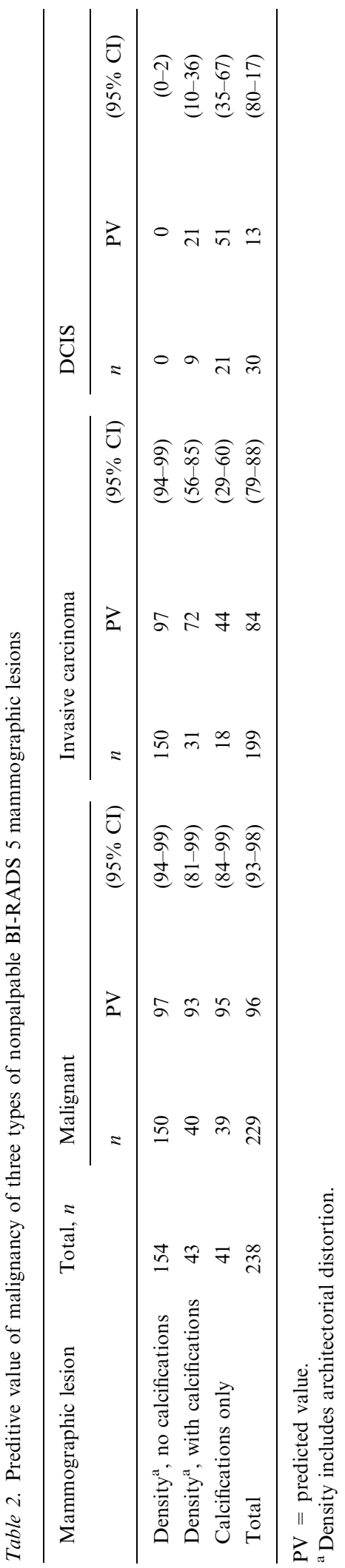

Proposed scenarios and results are based on the BI-RADS classification for various types of mammographic lesions and the cancer risk linked to it. Therefore, in practice, the prevalence of cancer in the subgroups should be assessed. This may differ according to background of referral of the patient (e.g., a higher cancer prevalence in patients referred through the national screening program compared to women visiting a breast cancer clinic for another reason) [15], type of hospital (e.g., a higher cancer prevalence in specialized cancer centers) or between countries (e.g., a higher cancer prevalence in Europe compared to the USA since the threshold for referral of malignancies in women with a suspicious nonpalpable breast lesion is lower in the USA) [16].

A second limitation is the reproduciblility of the BI-RADS classification. Variability in the classification of lesions according to the BI-RADS lexicon has been reported [17-20]. Specifically, the agreement between radiologists in reporting the presence of a finding is lower when cancer is present $(\kappa=0.54)$ compared to when cancer is not present $(\kappa=0.62)$ [20]. Since most BI-RADS 5 lesions represent cancer, it is clear that this influences further diagnostic and treatment decisions; thus cancer yield should be assessed in one's population of BI-RADS 5 lesions before using proposed scenarios.

An argument in favour of a preoperative diagnosis is that it reduces the occurrence of close or positive surgical margins. In our series where all patients first underwent SLCNB, we found that $\sim 23 \%$ of the local excision specimens (36/159) contained dubious or positive surgical margins. While some authors have reported lower rates of positive surgical margins at surgical excision after a core biopsy diagnosis (0-29\%) compared with diagnostic surgical excision biopsy [21-23] others have found margin positivity rates to be similar between the two diagnostic methods [24,25]. For BI-RADS 5 lesions, these rates have not been reported thusfar.

We do want to emphasize however, that taking a diagnostic surgical excision biopsy of a somewhat suspicious nonpalpable breast lesion is different compared to a surgical excision biopsy of a lesion that has $97 \%$ of being invasive cancer. In the latter example, the surgeon, aware of the potential presence of invasive disease, may excise a larger tissue volume, aiming for clear excision margins. 
Finally, when the preferred method of treatment for a patient is a mastectomy - if the suspicious lesions should be cancer - we recommend that a preoperative core needle biopsy diagnosis is obtained in all cases. Performing a mastectomy without a tissue diagnosis of cancer is obviously not recommended.

In conclusion, $96 \%$ of BI-RADS 5 lesions are malignant. Taking into account that as many of $97 \%$ of BI-RADS 5 lesions characterized by a mammographic density without calcifications turn out to be invasive cancer, surgical excision with sentinel node biopsy may be performed as the first diagnostic (and therapeutic) procedure in these cases, while SLCNB is the preferred diagnostic approach in all other cases.

\section{References}

1. Verkooijen HM, for the COBRA study group: Diagnostic accuracy of stereotactic large-core needle biopsy for nonpalpable breast disease: results of a multicenter prospective study with $95 \%$ surgical confirmation. Int $\mathbf{J}$ Cancer 99(6): 853-859, 2002

2. Fahy BN, Bold RJ, Schneider PD, Khatri V, Goodnight JE, Jr: Cost-benefit analysis of biopsy methods for suspicious mammographic lesions; discussion 994-5. Arch Surg 136(9): 990-994, 2001

3. D'Orsi CJ, Bassett LW, Feig SA: Illustrated Breast Imaging Reporting and Data System: Illustrated BIRADS. (3rd edn). American College of Radiology, Reston, VA, 1998

4. Buijs-van der Woude T, Verkooijen HM, Pijnappel RM, Klinkenbijl JH, Borel RI, Peeters PH, Buskens E: Cost comparison between stereotactic large-core-needle biopsy versus surgical excision biopsy in The Netherlands. Eur J Cancer 37(14): 1736-1745, 2001

5. Liberman L, Abramson AF, Squires FB, Glassman JR, Morris EA, Dershaw DD: The breast imaging reporting and data system: positive predictive value of mammographic features and final assessment categories. AJR Am J Roentgenol 171(1): 35-40, 1998

6. Orel SG, Kay N, Reynolds C, Sullivan DC: BI-RADS categorization as a predictor of malignancy. Radiology 211(3): 845-850, 1999

7. Lacquement MA, Mitchell D, Hollingsworth AB: Positive predictive value of the breast imaging reporting and data system. J Am Coll Surg 189(1): 34-40, 1999

8. Morrow M: When can stereotactic core biopsy replace excisional biopsy? - a clinical perspective. Breast Cancer Res Treat 36(1): 1-9, 1995

9. Margolin FR, Leung JW, Jacobs RP, Denny SR: Percutaneous imaging-guided core breast biopsy: 5 years' experience in a community hospital. AJR Am J Roentgenol 177(3): 559-564, 2001
10. Elvecrog EL, Lechner MC, Nelson MT: Nonpalpable breast lesions: correlation of stereotaxic large-core needle biopsy and surgical biopsy results. Radiology 188(2): 453455, 1993

11. Gisvold JJ, Goellner JR, Grant CS, Donohue JH, Sykes MW, Karsell PR, Coffey SL, Jung SH: Breast biopsy: a comparative study of stereotaxically guided core and excisional techniques. AJR Am J Roentgenol 162(4): 815-820, 1994

12. Ball CG, Butchart M, MacFarlane JK: Effect on biopsy technique of the breast imaging reporting and data system (BI-RADS) for nonpalpable mammographic abnormalities. Can J Surg 45(4): 259-263, 2002

13. Burrell HC, Pinder SE, Wilson AR, Evans AJ, Yeoman LJ, Elston CW, Ellis IO: The positive predictive value of mammographic signs: a review of 425 non-palpable breast lesions. Clin Radiol 51(4): 277-281, 1996

14. Lee CH, Egglin TK, Philpotts L, Mainiero MB, Tocino I: Cost-effectiveness of stereotactic core needle biopsy: analysis by means of mammographic findings. Radiology 202(3): 849-854, 1997

15. Hoorntje LE, Peeters PH, Borel Rinkes IH, Verkooijen HM, Pijnappel RM, Mali WP: Stereotactic large core needle biopsy for all nonpalpable breast lesions? Breast Cancer Res Treat 73: 177-182, 2002

16. Verkooijen HM, Peeters PH, Buskens E, Koot VC, Borel Rinkes IH, Mali WP et al.: Diagnostic accuracy of largecore needle biopsy for nonpalpable breast disease: a metaanalysis. Br J Cancer 82: 1017-1021, 2000

17. Baker JA, Kornguth PJ, Floyd CE, Jr: Breast imaging reporting and data system standardized mammography lexicon: observer variability in lesion description. AJR Am J Roentgenol 166(4): 773-778, 1996

18. Berg WA, Campassi C, Langenberg P, Sexton MJ: Breast imaging reporting and data system: inter- and intraobserver variability in feature analysis and final assessment. AJR Am J Roentgenol 174(6): 1769-1777, 2000

19. Berg WA, D'Orsi CJ, Jackson VP, Bassett LW, Beam CA, Lewis RS, Crewson PE: Does training in the breast imaging reporting and data system (BI-RADS) improve biopsy recommendations or feature analysis agreement with experienced breast imagers at mammography? Radiology 224(3): 871-880, 2002

20. Kerlikowske K, Grady D, Barclay J, Frankel SD, Ominsky SH, Sickles EA, Ernster V: Variability and accuracy in mammographic interpretation using the american college of radiology breast imaging reporting and data system. J Natl Cancer Inst 90(23): 1801-1809, 1998

21. Yim JH, Barton P, Weber B, Radford D, Levy J, Monsees B, Flanagan F, Norton JA, Doherty GM: Mammographically detected breast cancer. Benefits of stereotactic core versus wire localization biopsy. Ann Surg 223(6): 688-697, 1996

22. Lind DS, Minter R, Steinbach B, Abbitt P, Lanier L, Haigh L, Vauthey JN, Russin M, Hackett R, Copeland EM: Stereotactic core biopsy reduces the reexcision rate and the cost of mammographically detected cancer. J Surg Res 78(1): 23-26, 1998

23. Kaufman CS, Delbecq R, Jacobson L: Excising the reexcision: stereotactic core-needle biopsy decreases need 
170 LE Hoorntje et al.

for reexcision of breast cancer. World J Surg 22(10): 10231027, 1998

24. Liberman L, LaTrenta LR, Dershaw DD, Abramson AF, Morris EA, Cohen MA, Rosen PP, Borgen PI: Impact of core biopsy on the surgical management of impalpable breast cancer. AJR Am J Roentgenol 168(2): 495-499, 1997

25. Al Sobhi SS, Helvie MA, Pass HA, Chang AE: Extent of lumpectomy for breast cancer after diagnosis by stereo- tactic core versus wire localization biopsy. Ann Surg Oncol 6(4): 330-335, 1999

Address for offprints and correspondence: Dr L.E. Hoorntje, MD, PhD, St. Antonius Hospital, Department of Surgery, Koekoekslaan 1, 3435CM, Nieuwegein, The Netherlands; Tel.: +31-30-609 9111; Fax: +31-30-254 1944; E-mail:1_hoorntje@ yahoo.com 